and wood products still account for about one half of the exports. Metal and construction industries have followed in importance. Light industries, such as electronics, have more recently emerged as major contributors to employment and trade.

As a result of the industrial structure, physics has mostly been used in assistive roles, such as instrumentation, process control and quality assurance. On a smaller scale, however, more directly physics related industries have come forward. As examples can be cited the fabrication of meterological radiosondes, medical, environmental monitoring and nuclear instruments. For success in such efforts, international competitiveness has been required, since domestic markets are too small to support them.
The limited domestic energy sources, mostly in the form of hydropower and wood and peat reserves, and the large energy needs of heavy industries and domestic heating have encouraged Finland to seek new energy sources, in particular, extensive use of nuclear power. One pressurized water reactor of $440 \mathrm{MW}$ electric power was successfully put into operation in early 1977 and another is under construction on the southern coast at Loviisa. These reactors have been supplied by the Soviet Union to the state owned power company, which in collaboration with other suppliers has incorporated in the plant design the required containment and safety systems. Two boiling water reactors of $660 \mathrm{MW}$ electric power are in an advanced construction phase on the western coast at Olkiluoto. The units are of Swedish design and will be operated by a company owned by Finnish industries.

The development of nuclear power has benefitted from international collaboration through the International Atomic Energy Agency and the Nuclear Energy Agency of the OECD, as well as through several bilateral and multilateral agreements.

International contacts, whether in academic or applied physics are vital for a prosperous development of physics in a small country like Finland. The contributions of the European Physical Society in advancing such contacts are recognized and we hope that the meeting of the EPS Council in Helsinki will promote these aims on a wider European level.

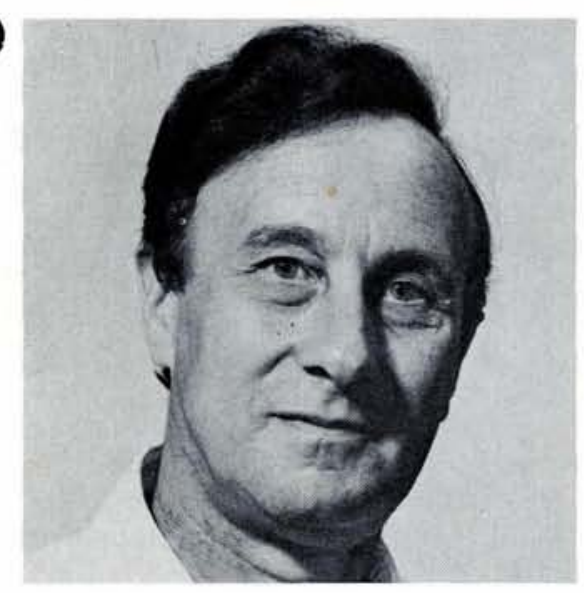

During recent years there has been a rapidly growing interest in the electronic properties of amorphous (a-) solids $\left.{ }^{1}\right)$. These materials have provided the solid state physicist with new and challenging fundamental problems and it is also likely that applications will follow the basic advances that have been made. The elemental a-semiconductors, $\mathrm{Si}$ and $\mathrm{Ge}$, which are the subjects of this article, have played a prominent part in this development. In the following sections I should like to give a brief outline of their preparation and electronic properties and then show how substitutional doping in the a-phase has opened up a wide range of new possibilities for both fundamental studies and device applications of a-semiconductors.

Structurally, a-materials lack the long range order found in the crystalline state, but in $\mathrm{a}-\mathrm{Si}$ and $\mathrm{Ge}$ the short range order is still determined by the well known tetrahedral bonds. $\mathrm{X}$-ray work on these materials has shown that at least some ordering can

\title{
Doped Amorphous Semiconductors
}

\section{W.E. Spear, Dundee Winner of the EPS 1977 Hewlett-Packard Europhysics Prize}

be found within a few atomic spacings around a given atom; it disappears at larger distances, mainly as a result of fluctuations in the bond angles. This has led to the concept of the "continuous random network" as a structural model characteristic of the a-state.

\section{Preparation of a-Semiconductors}

Probably the most widely used methods for depositing a-semiconductor films are evaporation in a high vacuum and cathodic sputtering. A striking feature of a-Si or a-Ge specimens prepared by these methods is the critical dependence of their electrical and optical properties on the experimental conditions during deposition and on the subsequent annealing treatment. It is now known that this behaviour is caused mainly by defect states in the mobility gap of the a-semiconductor. The random network in evaporated or sputtered specimens is far from continuous - it contains numerous vacancy-like defects which can form clusters of up to
$10 \AA$ across. Unsaturated (or "dangling") bonds at internal surfaces of the voids give rise to electronic states which often dominate the observed properties of the material and also introduce a marked sensitivity to reactive gases.

A basically different approach to preparation, which has played an important role in the development of the subject, has been the deposition of a-semiconductor films from the gas phase by decomposing the hydride in a radio frequency glow discharge. For instance, a-Si specimens are formed in this way from silane gas $\left(\mathrm{SiH}_{4}\right)$, a-Ge from germane, a-C from acetylene, and compounds such as a-SiC can be prepared from suitable gas mixtures. As we shall see, the electronic properties of glow discharge specimens are determined to a much lesser extent by the defect states mentioned above, so that experimental results are more representative of the intrinsic properties of the continuous random network. It is likely that gas phase deposition techniques will find 
increasing application, particularly for the preparation of doped a-semiconductors, and some of their main aspects will now briefly be discussed.

It had long been recognised that a glow discharge plasma provides a useful means of promoting chemical reactions at comparatively low ambient temperatures. For instance, plasma techniques are being currently used in the electronics industry for photoresist removal and etching. The deposition of an a-semiconductor with reproducible and optimised electronic properties is, however, a far more critical application of the glow discharge technique. Figs. 1(a) and 1(b) show the general lay-out of two experimental arrangements. The first, originally described by Sterling and his collaborators ${ }^{2}$ ) has been used in most of our earlier work on a-Si. The gas, G, flows through the quartz reaction tube, $\mathrm{T}$, past the substrate, $\mathrm{S}$, which is held at a temperature of about $250^{\circ} \mathrm{C}$ on the pedestal, $H$. The plasma, $P$, is maintained by predominantly inductive coupling of RF power into the gas. The power level is small, typically 10-20 W, and frequencies between 1 and $100 \mathrm{MHz}$ have been tried. The capacitatively coupled arrangement shown in Fig. 1(b) is now being used by a number of laboratories for depositing larger area specimens. The apparent simplicity of the technique is deceptive. Complex reactions take place in the plasma at the surface of the growing semiconductor film. Their accurate control, which is essential for optimised electronic properties, still presents many problems.

\section{Electronic States and Transport in an a-Semiconductor}

Fig. 2 shows a typical density of state distribution of an a-semiconductor. The function $g(\varepsilon)$ denotes the number of states per unit volume in unit energy interval; it is plotted here

Fig. 2. Schematic density of state distribution expected for an a-semiconductor. $g(\varepsilon)$, the number of states per unit volume and energy interval is plotted on a logarithmic scale against the energy $\varepsilon$. $E$, extended states ; $T$, band tail states ; $G$, gap states.

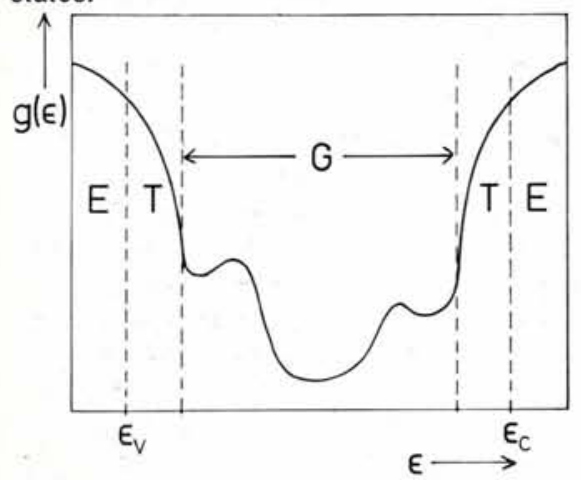

logarithmically against the electron energy $\varepsilon$. As indicated, the energy spectrum can be divided according to the electronic character of the states into extended states $E$, band tail states $T$, and gap states $G$.

First consider the extended states in the energy ranges $\varepsilon>\varepsilon_{c}$ for electrons and $\varepsilon<\varepsilon_{y}$ for holes, which in the a-material correspond to the crystalline conduction at valence bands. As one approaches $\varepsilon_{c}$ or $\varepsilon_{v}$ the effects of random potentials and of fluctuations in the interatomic distances and bond angles begin to dominate the transport. In the case of electrons, the mean free path just above $\varepsilon_{c}$ and the coherence length of the electron wave functions approach the interatomic separation. Transport just above $\varepsilon_{c}$ can no longer be regarded as band motion with occasional scattering as is done in crystalline transport theory. In this borderline region, conduction is essentially a diffusive process, quite similar to Brownian motion. The electron can be envisaged as tunnelling from site to site with an electronic frequency but without any thermal activation.

Below $\varepsilon_{c}$, localisation sets in. This change in the nature of the electron wavefunctions in the band tail states is of fundamental importance to the understanding of the non-crystalline state $\left.{ }^{3}\right)$. Localisation is a direct consequence of the increasing disorder potential and means that the electrical conductivity associated with these states vanishes as the absolute zero temperature is approached. The tail states arise from the absence of long range order and are a feature of the continuous random network; the extent of the distribution is an approximate measure of the disorder potential. On the other hand, the deeper lying gap states, G, in a tetrahedral a-semiconductor are determined not by the disorder, but by structural defects in the random network. Their electronic structure appears to be sufficiently well defined within the existing short range ordar to lead to recognisable features in $g(\varepsilon)$. In the undoped material, the position of the Fermi level $\varepsilon_{f}$ is determined by the charge distribution in the gap states.

The density of states $g(\varepsilon)$ in the mobility gap of a-Si has been investigated in considerable detail by the Dundee group using field effect techniques $4,5,6)$. The results show clearly the presence of tail and gap states discussed above. The most significant feature of this work is however the information it provides on the strong dependence of $g(\varepsilon)$ on the method of
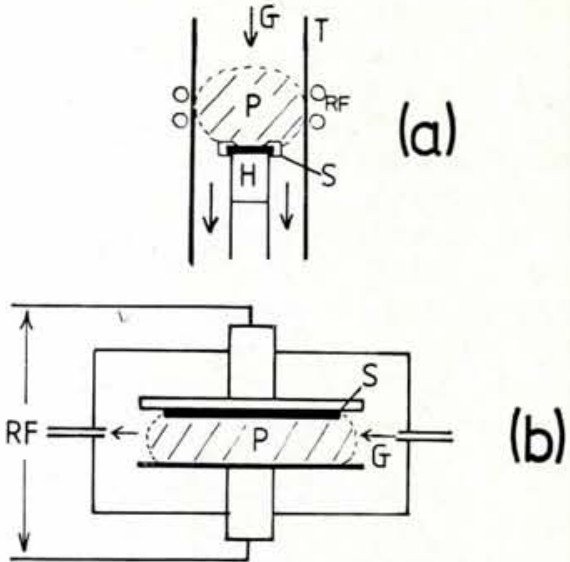

Fig. 1. Experimental methods for gas phase deposition of an a-semiconductor (a) inductive coupling to RF (b) capacitative coupling.

preparation and the preparation conditions. In particular, it was found that a-Si specimens deposited by the glow discharge technique possessed a remarkably low overall density of gap states $\left(\mathrm{g}\left(\varepsilon_{\mathrm{f}}\right) \cong 10^{17} \mathrm{~cm}^{-3} \mathrm{eV}^{-1}\right)$, up to two orders of magnitude lower than that in specimens produced by vacuum evaporation or cathodic sputtering $\left.{ }^{5}\right)$. These results originally suggested that glow discharge a-Si might be a most suitable material for efficient doping in the a-phase.

The electronic transport in a-Si has been studied extensively by conductivity ${ }^{7}$ ), drift mobility 7,8 ), thermoelectric power ${ }^{9}$ ) and Hall effect ${ }^{10}$ ) measurements. In undoped glow discharge specimens, the conduction above about $250 \mathrm{~K}$ takes place in the extended electron states. Below this temperature the predominant current path moves into the electron tail states, where a new transport mechanism, phonon-assisted hopping, takes over. Hopping mobilities in this energy range are $10^{-2} \mathrm{~cm}^{2} \mathrm{~V}^{-1} \mathrm{~s}^{-1}$ or less ${ }^{7}$ ), several orders of magnitude smaller than the mobility in the extended states. The drastic change in transport properties at $\varepsilon_{c}$ (and $\varepsilon_{v}$ ) defines the so-called mobility gap of the a-material.

\section{Doped a-Semiconductors}

Substitutional doping of a semiconductor such as $\mathrm{Si}$ or $\mathrm{Ge}$ mieans that an atom of the tetrahedrally bonded structure has been replaced by a pentavalent or trivalent impurity atom such as phosphorus (donor) or boron (acceptor). In crystalline semiconductors, this possibility has been one of the most important factors in the development of semiconductor physics and of solid state electronics. With the growing interest in a-semiconductors, attempts have been made in recent years to control and modify 
Fig. 3. Room temperature conductivity $\sigma_{R T}$ of $n$ - and p-type amorphous Si specimens, plotted as a function of the gaseous impurity ratio. For the right-hand curve, this is the number of phosphine to silane molecules in the gas mixture used for specimen preparation. On the left it is the corresponding diborane to silane ratio. The centre refers to undoped specimens. (After ref. 12)

the electrical properties of such films by doping with the normal substitutional impurities used in the crystals. However, the results of these experiments led to the conclusion that evaporated or sputtered film of a-Si or a-Ge cannot be doped effectively.

This basic limitation was overcome in 1975 when Spear and Le Comber showed $11,12,13$ ) that substitutional doping of a-Si and a-Ge is possible from the gas phase and that it can provide systematic control of the electronic properties over a remarkably wide range. This is achieved in the case of a-Si by adding small, but accurately determined amounts of phosphine or diborane (the hydrides of phosphorus and boron) to the silane flowing through the apparatus shown in Fig. 1. In the preparation units developed in our laboratory, the doped gases are pre-mixed and kept in reservoirs, so that in principle any desired sequence of $n$ - and p-type layers can be deposited by opening or closing the appropriate taps.

Fig. 3 illustrates the wide-ranging control that can be obtained by this approach ${ }^{12}$ ). The room temperature conductivity, $\sigma_{\mathrm{RT}}$, of a series of $\mathrm{n}$ - and p-type a-Si specimens is plotted against the gaseous impurity ratio. On the right hand side this is the ratio $\mathrm{N}\left(\mathrm{PH}_{3}\right) / \mathrm{N}\left(\mathrm{SiH}_{4}\right)$, the number of phosphine to silane molecules in the gaseous mixture, whereas on the lefi the corresponding diborane to silane ratio is shown. In the centre of the graph, the conductivities of $10^{-8}$ to $10^{-9}$ $(\Omega \mathrm{cm})^{-1}$ are representative of undoped glow discharge specimens.

Consider first the $n$-side. The encouraging feature is that even a minute quantity of phosphine, $6 \times 10^{-6}$ ppv, increases $\sigma_{\text {RT }}$ by over two orders of magnitude. This clearly supports deductions from field effect measurements concerning the low value of $g\left(\varepsilon_{f}\right)$. With a phosphine to silane ratio of $10^{-3}$ one approaches $\sigma_{\mathrm{RT}} \cong 10^{-2}$ $(\Omega \mathrm{cm})^{-1}$; that is, $\sigma_{\mathrm{RT}}$ has been increased by seven orders of magnitude by phosphine doping.

Turning now to the effect of boron doping, we see that initially $\sigma_{\mathrm{RT}}$ decreases to about $10^{-12}(\Omega \mathrm{cm})^{-1}$. This feature can be understood from the density of state distribution. At first

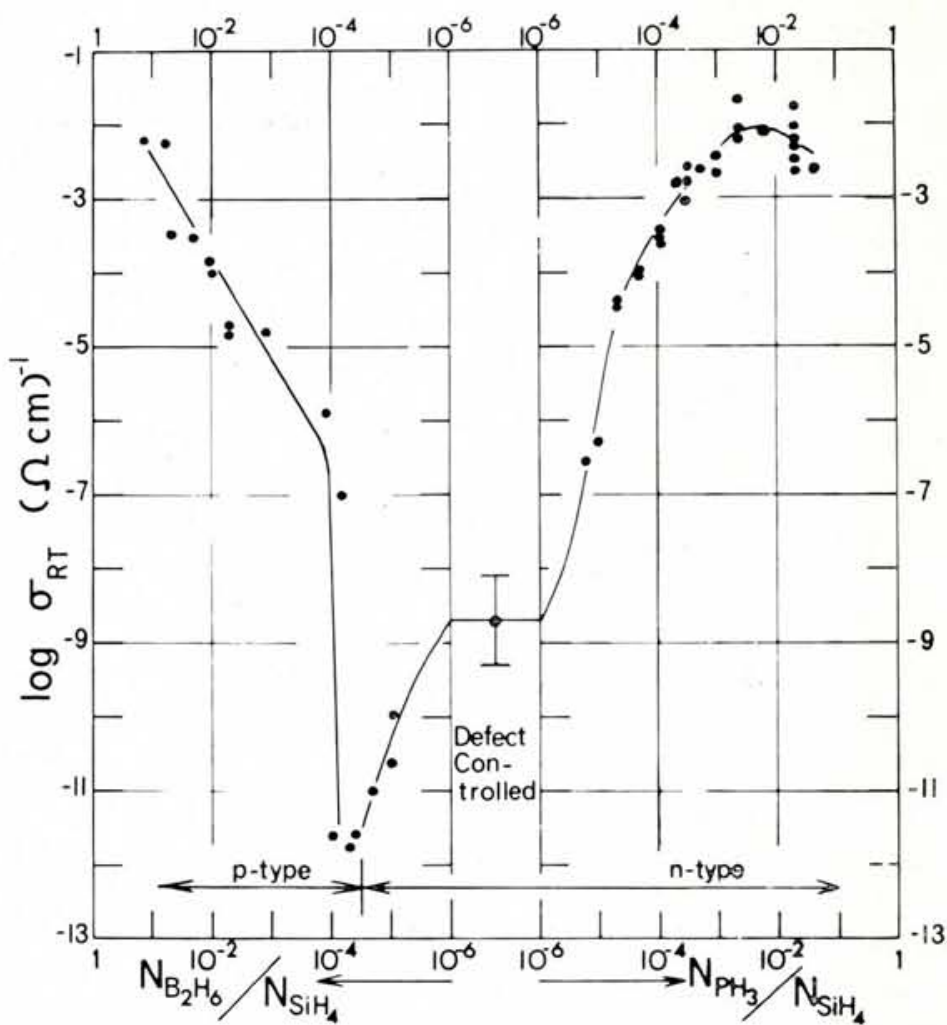

$\varepsilon_{\mathrm{f}}$ is moved to the p-side of the density of states minimum and the decreasing $\sigma$ reflects the rapid reduction in the number of electrons at $\varepsilon$ At a diborane to silane ratio of $10^{-4}$ there occurs an almost vertical rise in $\sigma_{R T}$ by six orders of magnitude, indicating that hole conduction has taken over. The complete doping range corresponds to a movement in $\varepsilon_{\mathrm{f}}$ of $1.2 \mathrm{eV}$, essentially between the onset of the electron and hole tail states. It would be difficult to explain these results other than by a model of substitutional impurities, which in most respects is similar to that used for crystalline materials. Yet, it is important to realise that the effect of ionised donors or acceptors on the electrical properties of an a-semiconductor is basically different from that in the crystalline material. In the latter $\mathrm{N}_{\mathrm{D}}^{+} \cong \Delta \mathrm{n}\left(\varepsilon_{\mathrm{c}}\right)$, the increase in electron density in the con-

duction band (exhaustion range). In the amorphous case, changes in electrical properties are brought about primarily by changes in the gap state occupation. For sensitive doping $\Delta \varepsilon_{f}$ which is of the order of $\mathrm{N}_{\mathrm{D}} / \mathrm{g}\left(\varepsilon_{1}\right)$, should be as large as possible; a low level of gap states is therefore an essential condition.

In the deposition of a-Si, the glow discharge plasma contains both hydrogen and silicon-hydrogen ions. It has often been argued that the low overall $\mathrm{g}(\varepsilon)$ in glow discharge specimens simply results from saturating the dangling bonds in the gap states by hydrogen; the extremely high hydrogen content found in some a-Si specimens 1) has been considered as convincing evidence for this view. In the author's opinion an equally important reason may be the plasma deposition itself, in which the film grows

\section{POST DOCTORAL RESEARCH POSITIONS \\ POST-DOCTORAL RESEARCH POSITIONS AT \\ SWISS FEDERAL INSTITUTE OF TECHNOLOGY ZURICH \\ Two post-doctoral research positions are available at: the Laboratory for High Energy Physics. \\ Candidates should be interested in muonic and pionic atom research being conducted at the Swiss Institute of Nuclear Research (SIN). Present experiments are on muonic X-ray measurements with a curved-crystal spectrometer. Applicants are expected to have experience in medium-energy physics. A three year appoint- ment is offered, with possible renewal. Permanency could be considered for one of the positions later. \\ Applicants are invited to submit their résumé, the list of publications and the names of two referees to: \\ Professor H.J. Leisi, Laboratory for High Energy Physics ETHZ, c/o SIN, $\mathrm{CH}$ - 5234 Villigen (Switzerland).}


under conditions basically different from those achievable by other techniques, so that density and size of defects may be appreciably smaller. $\mathrm{Ne}$ vertheless, the recent work of Paul and his collaborators ${ }^{14}$ ) has shown convincingly that the presence of hydrogen during sputtering of a-Si reduces $g(\varepsilon)$ to a sufficiently low level that doping now becomes possible.

Finally, it is of interest to note that attempts to dope a-Si by ion implantation have led to encouraging results ${ }^{15}$ ). The doping range that can be covered is the same as in the gas phase, (Fig. 3), but the doping efficiency is considerably less.

\section{Recent Developments and Possible Applications}

There is no doubt that the new field of doped a-semiconductors has opened up exciting possibilities for the study of these materials and for their application in cheap, large-area thin film devices. Many research groups have entered the field and, as the Proceedings of the 1977 International Conference ${ }^{1}$ ) show, progress is being made on both the fundamental and applied sides.

Recently the transport properties of n-type a-Si specimens have been investigated as a function of donor density ${ }^{8}, 9,{ }^{10}$ ). When $N_{D}$ exceeds about $10^{18} \mathrm{~cm}^{-3}$, a new conduction path is established in the electron tail states in which the electrons hop between the donor sites. By varying the average hopping distance through doping, the extension of the phosphorus donor wavefunctions can be determined. The value of $22 \AA$ is closely similar to that in crystalline Si.

Of all the transport measurements, the Hall effect in a-solids differs most significantly from that in crystals, where it generally provides clear-cut and easily interpretable information. The main reason is the extremely short mean free path of carriers in the extended states of the a-material. Theo$\mathrm{ry}^{16}$ ) shows that in this case the Hall mobility should depend on the configuration of tunnelling sites and on the electron transfer energy between sites; it should also be temperature independent and have a magnitude approximately $1 / 10$ of the drift mobility. Measurements on lightly doped n-type a- $\mathrm{Si}^{10}$ ) are in substantial agreement with the last two predictions. However, the most intriguing result is the double reversal in the sign of the Hall coefficient: phosphorus doped specimens, in which conduction is undoubtedly by electrons, give the sign expected for positive carriers, whereas in boron doped samples the sign corresponds to that for free electrons. The reasons for this anomaly are not entirely clear at present, but it is likely that the effect may be associated with the different nature of the orbitals in the valence and conduction bands.

Undoped a-Si is an efficient photoconductor ${ }^{17}$ ) with a response extending from the visible into the near-infrared spectral regions. Recent work on the recombination of photogenerated carriers in $\mathrm{a}-\mathrm{Si}^{18}$ ) has shown that slight phosphorus doping leads to a remarkable sensitisation in photoconductivity. The photoconductive gain increases by a factor of about 20 to values well above unity. It has been suggested that as the Fermi level is moved towards $\varepsilon$ by doping, recombination centres with comparatively large Coulomb cross-sections are being filled, which results in the observed increase in electron lifetime.

Perhaps the most promising applied development arising out of the a-Si work has been the amorphous $p-n$ junction. Early in 1976 we showed ${ }^{19}$ ) that an amorphous analogue of this basic solid state device could be prepared by the doping techniques described above. The thin film junction, about $1 \mu \mathrm{m}$ thick, consists of $\mathrm{p}$ - and n-type layers, sandwiched between evaporated metal electrodes. During the past year we have investigated the formation of an a-junction and measured its performance as a function of preparation conditions, doping levels and barrier profile. There is no doubt that the main features of crystalline $p-n$ junctions can be reproduced in the a-devices. However, a basic difference remains in the formation of the junctions, caused once again by the presence of gap states in the a-material. Whereas in the crystal, the space charge density in the junction region is determined entirely by the ionised donors or acceptors, in the a-case, the net charge density will depend both on ionised impurities and on the distribution of the gap states in energy. This can have pronounced effects on the barrier profile and on the differential capacity of the device.

The possible application of a-Si in cheap, large-area photovoltaic devices for solar energy conversion is at present attracting a great deal of interest. Last year Carlson and Wronski ${ }^{20}$ ) of the RCA Laboratories described an a-Si photovoltaic $p-i-n$ junction deposited by the glow discharge technique, as well as a Pt Schottky barrier cell with a conversion efficiency of $5,5 \%{ }^{21}$ ). This represents a promising start and it is reasonable to hope that viable efficiencies of the order of $10 \%$ will be attained within the next few years. At present the most serious limitations are the short minority carrier lifetimes ( $\leqslant 0.3 \mu \mathrm{s})$ and a systematic effort to improve material preparation techniques is needed to overcome this problem. If successful, the decisive factor in the photovoltaic development will ultimately be the cost per $\mathrm{kW}$ of power. The present figure of $\$ 15,000-\$ 20,000$ for crystalline Si arrays severely restricts their application on purely economic grounds. It is difficult at this stage to predict the eventual cost of a-Si devices, but it may be possible to cut the above figure for crystalline arrays by a factor of 40-50 in the large scale production of amorphous photovoltaic panels. In that case, a-Si devices could make a useful contribution to future energy needs.

\section{References}

1. See for instance: Proceedings of the 7th International Conference on Amorphous and Liquid Semiconductors, ed. by W.E. Spear (CICL, University of Edinburgh) 1977

2. CHITTICK, R.C., ALEXANDER, J.H. and STERLING, H.F. J. Electrochem. Soc. 116 (1969) 77

3. MOTT, N.F. Phil. Mag. 17 (1968) 1259 ; ibid. 19 (1969) 835 and J. Non-Cryst. Solids 8-10 (1972) 1 ; see also MOTT, N.F, and DAVIS, E.A. Electronic Processes in Non-Crystalline Materials (University Press, Oxford) 1971

4. SPEAR, W.E. and LE COMBER, P.G. J. Non-Cryst. Solids 8-10 (1972) 727

5. MADAN, A., LE COMBER, P.G. and SPEAR, W.E. J. Non-Cryst. Solids 20 (1976) 239

6. MADAN, A. and LE COMBER, P.G. Ref. 1 p. 377

7. LE COMBER, P.G., MADAN, A. and SPEAR, W.E. J. Non-Cryst. Solids 11 (1972) 219

8. ALLAN, D., COMBER, P.G. and SPEAR, W.E. Ref. 1 p. 323

9. JONES, D.I., LE COMBER, P.G. and SPEAR, W.E. Phil. Mag. 36 (1977) 541

10. LE COMBER, P.G., JONES, D.I. and SPEAR, W.E. Phil. Mag. 35 (1977) 1173

11. SPEAR, W.E. and LE COMBER, P.G. Solid State Comm. 17 (1975) 1193

12. SPEAR, W.E. and LE COMBER, P.G. Phil. Mag. 33 (1976) 935

13. SPEAR, W.E. Advances in Physics January, 1978

14. PAUL, W., LEWIS, A.J., CORNELL, G.A.N. and MOUSTAKAS, T.D. Solid State Comm. 20 (1976) 969

15. MÜLLER, G., KALBITZER, S., SPEAR, W.E. and LE COMBER, P.G. Ref. 1 p. 442

16. FRIEDMEN, L. J. Non-Cryst. Solids 6 (1971) 329

17. LOVELAND, R.J., SPEAR, W.E. and ALSHARBATY, A. J. Non-Ciyst. Solids 13 $(1973-4) 55$

18. ANDERSON, D.A. and SPEAR, W.E. Phil. Mag. 36 (1977) 695

19. SPEAR, W.E., LE COMBER, P.G., KINMOND, S. and BRODSKY, M.H. Appl. Phys. Letters 28 (1976) 105

20. CARLSON, D.E. and WRONSKI, C.R. Appl. Phys. Letters 28 (1976) 671

21. CARLSON, D.E. IEEE Trans. on Electron Devices 24 (1977) 449 